\title{
Higgs results from the ATLAS experiment (Standard Model and Beyond)*
}

\author{
Camila Rangel Smith
}

Uppsala University

\begin{abstract}
The discovery of a new particle consistent with the SM-like Higgs boson, with a mass of about $125 \mathrm{GeV}$, was announced by the ATLAS and CMS experiments on the 4 July 2012. After the discovery, the properties of this new particle are studied with high precision measurements of its mass, spin and couplings in all the accessible decay channels. In this document, recent results from the ATLAS experiment in this topic are presented, including: $H \rightarrow \gamma \gamma, H \rightarrow Z Z^{(*)} \rightarrow 4 l, H \rightarrow W W^{(*)} \rightarrow l \nu l \nu, H \rightarrow \tau \tau, H \rightarrow Z \gamma$, $H \rightarrow \mu \mu V H \rightarrow b \bar{b}, V H \rightarrow W W^{(*)}, t t H \rightarrow \gamma \gamma$. Furthermore, searches for beyond the standard model Higgs bosons are exposed.
\end{abstract}

PACS numbers: 14.80.Bn, 14.80.Ec, 14.80.Fd

\section{Introduction: Higgs boson physics}

In the SM the Higgs boson couples preferentially to heavy particles, such as the $\mathrm{Z}$ boson, the $\mathrm{W}$ boson, and the top quark. Thus, there are mainly four production models for the SM in proton-proton collisions:

- The dominant is gluon-gluon fusion (ggF), where the Higgs boson couples indirectly to gluons via a triangular loop of quarks dominated by the top.

- The Vector-boson fusion (VBF), in which the Higgs boson is produced by the fusion of two weak vector bosons radiated from quarks. The two final quarks fragment into two forward jets, and no QCD activity is expected between the two jets where the Higgs decay products are, leaving a special signature in the detector that can be used to suppress the QCD background.

\footnotetext{
* Presented at Epiphany 2014
} 
- The associated production with vector bosons is called Higgsstrahlung (WH or $\mathrm{ZH})$. It is an interesting mode to study the couplings to the vector bosons.

- The Higgs production in association with top pairs (ttH) is the smallest mode contributing in the LHC Higgs production. Nevertheless, is an important process to measure the Yukawa coupling between the Higgs and the top quark.

The partial decay width of the Higgs boson depends on the mass of the decay products. In ATLAS a large number of exclusive decays of the Higgs boson are studied to determine the nature of this new particle. The most relevant channels are described in the next sections.

\section{The $H \rightarrow \gamma \gamma$ channel}

The di-photon channel was the first to observe the Higgs boson. The analysis strategy is based on the di-photon invariant mass $\left(m_{\gamma \gamma}\right)$ as a main discriminant variable, built by a photon pair with well measured energies and directions. For the search, the $m_{\gamma \gamma}$ range is scanned with the objective of finding a resonance peak, over a large QCD combinatorial background. The background is composed of pairs of photons, associated production of photons with jets, and processes with several jets in the final state. To increase the analysis sensitivity, the events are separated into different categories with different mass resolutions and signal to background ratios. An extended unbinned maximum-likelihood fit is performed simultaneously to all categories to extract the signal and background event yields and determine the significance of the signal.

The analysis uses the complete dataset of the first LHC run of corresponding to an integrated luminosity of $20.7 \mathrm{fb}^{-1}$ recorded at $\sqrt{s}=8 \mathrm{TeV}$ and $4.7 \mathrm{fb}^{-1}$ at $\sqrt{s}=7 \mathrm{TeV}$. In Figure 1 , the inclusive invariant mass distribution of the di-photon candidates is shown, overlaid with the signalplus-background fit. The largest local signal significance in the combined data sample is found to be $7.4 \sigma$ at a mass $\left(m_{H}\right)$ of $126.5 \mathrm{GeV}$, where the expected significance is $4.1 \sigma$ [1].

\section{The $H \rightarrow Z Z^{(*)} \rightarrow 4 l$ channel}

The analysis uses a sample with an integrated luminosity of $20.7 \mathrm{fb}^{-1}$ recorded at $\sqrt{s}=8 \mathrm{TeV}$ and $4.7 \mathrm{fb}^{-1}$ at $\sqrt{s}=7 \mathrm{TeV}$. Events are required to have two pairs of same-flavour, opposite charge, isolated leptons: $4 e, 2 e 2 \mu$, $2 \mu 2 e, 4 \mu$. The largest background comes from di-boson $\mathrm{ZZ}^{(*)}$ production. Important contributions arise also from $\mathrm{Z}+$ jets and $t \bar{t}$ production. 


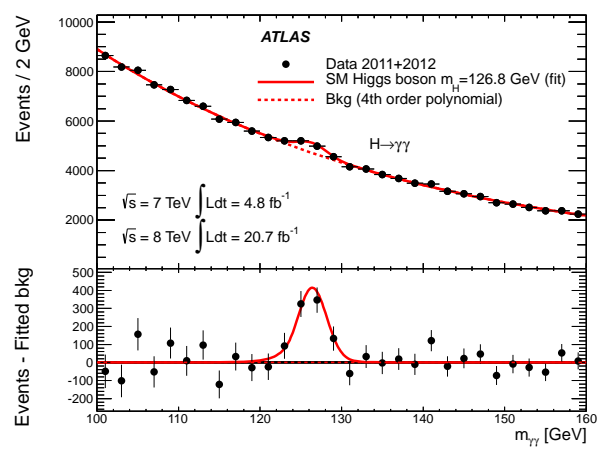

Fig. 1. Invariant mass distribution of di-photon candidates for the combined $7 \mathrm{TeV}$ and $8 \mathrm{TeV}$ data samples. The result of a fit to the data of the sum of a signal component fixed to an hypothesised Higgs mass of $126.8 \mathrm{GeV}$ and a background component described by a fourth-order Bernstein polynomial is superimposed. The bottom inset displays the residuals of the data with respect to the fitted background component [1].

The mass reconstruction uses a constrained fit to the $\mathrm{Z}$ mass to improve the resolution, and the final state radiation (FSR) is included in the reconstruction of the leading $\mathrm{Z}$ decaying into muons. Similar to the $H \rightarrow \gamma \gamma$ channel, events are categorised separating candidate events into ggF-like, $\mathrm{VBF}$ and VH like events.

The reconstructed four-lepton mass spectrum of the inclusive analysis is shown in Figure 2. A deviation from the background-only expectation is observed at a mass of $124.3 \mathrm{GeV}$, with a significance of $6.6 \sigma$ for the combined $7 \mathrm{TeV}$ and $8 \mathrm{TeV}$ data ( $4.4 \sigma$ expected, see Figure 3$)$. This result establishes a discovery-level signal in this channel alone [1].

\section{The $H \rightarrow W W^{(*)} \rightarrow l \nu l \nu$ channel}

The $H \rightarrow W W^{(*)} \rightarrow l \nu l \nu$ channel rate is large, but has a limited mass resolution due to the presence of two neutrinos in the final state. Events are required to have two opposite-charge leptons and a large missing energy from the neutrinos. The dominant SM backgrounds are $\mathrm{WW}^{(*)}, t \bar{t}$ and $\mathrm{Wt}$.

The events are categorised by jet multiplicity (Njets), which allows control of the background from top quarks (containing b-quark jets), as well as the extraction of the signal strengths for the ggF and VBF production processes.

The transverse mass distributions for Njet $\leq 1$ and Njet $\geq 2$ final states are shown in Figure 4 using a sample corresponding to an integrated lumi- 


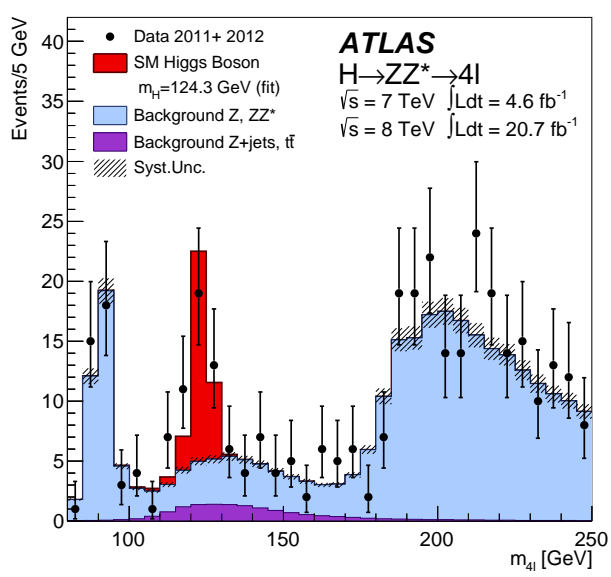

Fig. 2. The distribution of the four-lepton invariant mass, m4l, for the selected candidates in the data. The estimated background, as well as the expected SM Higgs boson signal for $\mathrm{mH}=124.3 \mathrm{GeV}$ (scaled by the signal strength obtained from fits to the data), are also shown [1].

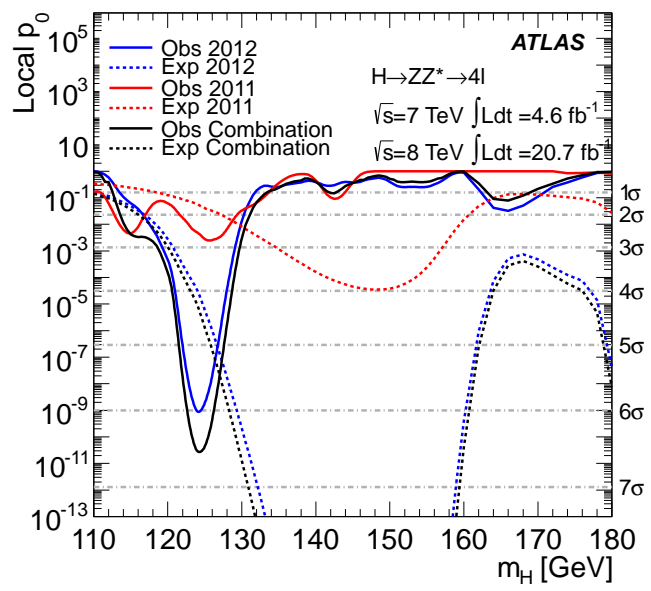

Fig. 3. The observed local p0 for the the $\sqrt{s}=7 \mathrm{TeV}$ data (red), the $\sqrt{s}=8 \mathrm{TeV}$ data (blue) and their combination (black). The dashed curves show the expected median local p0 for a SM Higgs boson hypothesis when tested at a given $\mathrm{mH}$ [1]. 

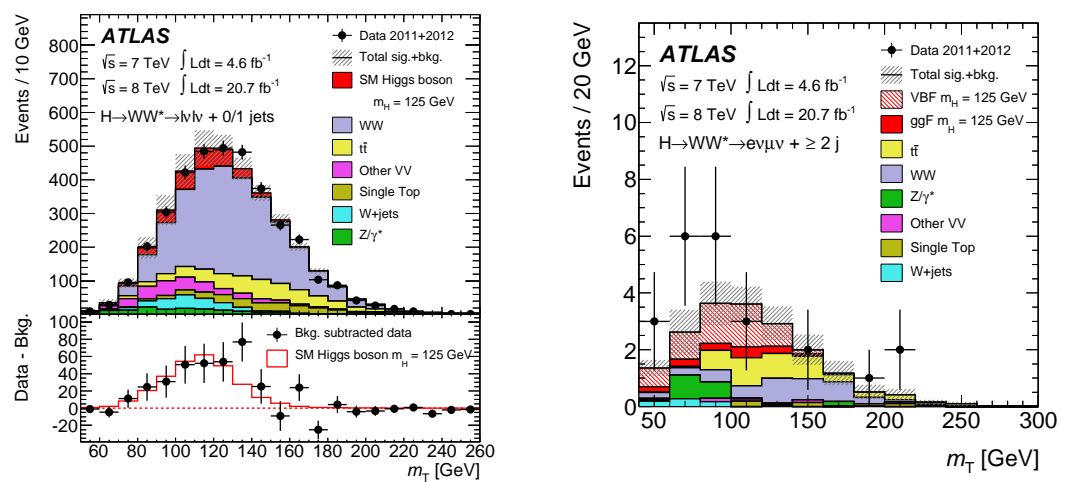

Fig. 4. Transverse mass distributions for events passing the full selection of the $H \rightarrow W W^{(*)} \rightarrow l \nu l \nu$ analysis: Left is summed over all lepton flavours for final states with $\mathrm{Njet} \leq 1$; on the right different-flavour final states with and Njet $\geq 2$ - The hatched area represents the total uncertainty on the sum of the signal and background yields from statistical, experimental, and theoretical sources [1].

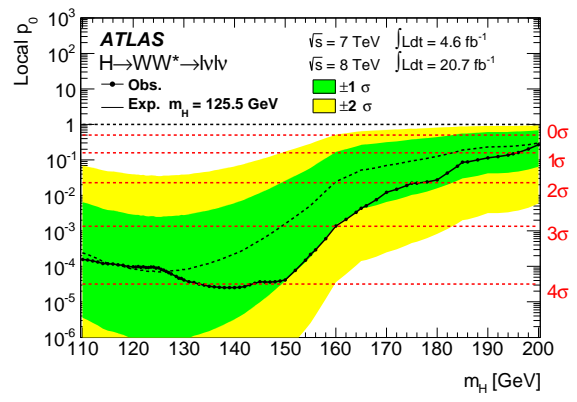

Fig. 5. The expected and observed local p0 values as a function of $m_{H}$ for the $H \rightarrow W W^{(*)} \rightarrow l \nu l \nu$ analysis of the combined $7 \mathrm{TeV}$ and $8 \mathrm{TeV}$ data. The green (yellow) band indicates the $\pm 1 \sigma( \pm 2 \sigma)$ uncertainty on the expected p0 curve [1].

nosity of $20.7 \mathrm{fb}^{-1}$ at $\sqrt{s}=8 \mathrm{TeV}$ and $4.7 \mathrm{fb}^{-1}$ at $\sqrt{s}=7 \mathrm{TeV}$. An excess of events is observed in the data with respect to the background-only expectation at $m_{H}=125.5 \mathrm{GeV}$, with an observed and expected significance of $3.8 \sigma$ for the SM Higgs boson [1] as shown in Figure 5.

\section{The $H \rightarrow \tau \tau$ channel}

The search is performed in all the $\tau$ decay combinations: $\tau l e p-\tau l e p$,

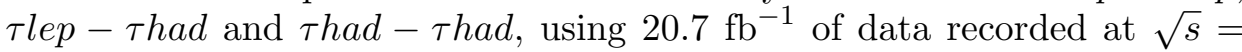




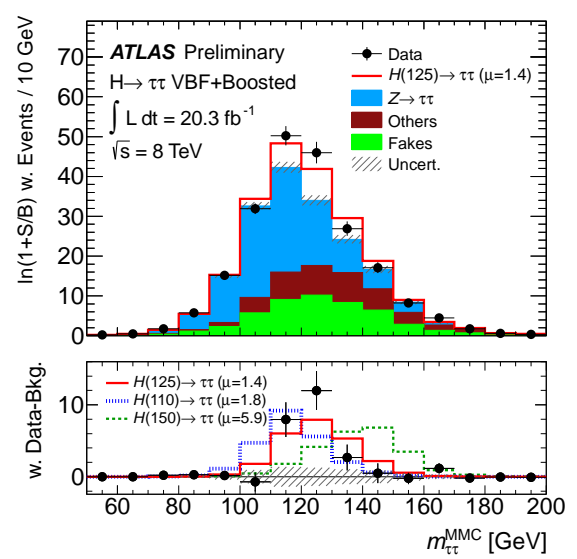

Fig. 6. Distribution for $m_{\tau \tau}^{M M C}$ where events are weighted by $\ln (1+\mathrm{S} / \mathrm{B})$ for all channels. These weights are determined by the signal and background predictions for each BDT bin. The bottom panel in each plot shows the difference between weighted data events and weighted background events (black points), compared to the weighted signal yields. The background predictions are obtained from the global fit with the $m_{H}=125 \mathrm{GeV}$ signal hypothesis $(\mu=1.4)$. The $m_{H}=125 \mathrm{GeV}$ signal is plotted with a solid red line with the signal strengths set to their best fit values [2].

$8 \mathrm{TeV}$. The dominating background is $Z \rightarrow \tau \tau$ (irreducible), following by the reducible $Z(e e / \mu \mu)+$ jets, $\mathrm{W}+$ jets, top, multi-jets and di-bosons. Each channel is affected differently by these backgrounds, therefore the event selections are optimised separately.

Two analysis categories are defined in an exclusive way: VBF, with the presence of two jets with a large pseudo-rapidity separation and boosted, which is targeted at events with a boosted Higgs boson from ggF (Higgs $\left.p_{T}>100 \mathrm{GeV}\right)$. The analysis strategy is based on a Boosted Decision Tree (BDT) used in each category to extract the Higgs signal from the large backgrounds.

A deviation from the background-only hypothesis is found with an observed (expected) significance of 4.1 (3.2) standard deviations, and a measured signal strength of $\mu=1.4+0.5-0.4$. The compatibility of this excess of events above background predictions, with the SM Higgs boson at $m_{H}=$ $125 \mathrm{GeV}$ is visualised with a weighted distribution of events as a function of $m_{\tau \tau}^{M M C}$ (MMC stands for Missing Mass Calculator. More information is found in [2]), shown in Figure 6. 


\section{Higgs properties measurements}

The results from the individual channels described in the previous sections are combined to extract information about the Higgs boson mass, production properties and couplings. Details of the statistical methods for the combination are found in [1]. A short summary of the results is presented next.

\subsection{Mass and signal strength}

The two channels with the best mass resolution are used for the mass determination: $H \rightarrow \gamma \gamma$ and $H \rightarrow Z Z^{(*)} \rightarrow 4 l$. In the two cases, $m_{H}=$ $126.8 \pm 0.2$ (stat) \pm 0.7 (sys) $\mathrm{GeV}$ and $m_{H}=124.3_{-0.5}^{+0.6}$ (stat) ${ }_{-0.3}^{+0.5}$ (sys) $\mathrm{GeV}$ are obtained from fits to the mass spectra.

The combined mass is measured to be:

$$
m_{H}=125.5 \pm 0.2(\text { stat })_{-0.6}^{+0.5}(\text { sys }) \mathrm{GeV},
$$

where the main sources of systematic uncertainty are the photon and lepton energy and momentum scale.

The Higgs boson production strength $\mu$ is measured from a fit to the data using the profile likelihood ratio for a fixed mass hypothesis corresponding to the measured combined value $m_{H}=125.5 \mathrm{GeV}$.

The signal production strength normalised to the SM expectation, obtained by combining the $H \rightarrow \gamma \gamma, H \rightarrow Z Z^{(*)} \rightarrow 4 l$ and $H \rightarrow W W^{(*)}$ channels, is:

$$
\mu=1.33 \pm 0.14 \text { (stat) } \pm 0.15 \text { (sys) }
$$

where the systematic uncertainty receives similar contributions from the theoretical uncertainty on the signal cross section and experimental sources. The production strengths measured in the three channels and in their main analysis categories are shown in Figure 7.

\subsection{Coupling measurements}

The measurements of couplings are implemented using a leading-order tree-level motivated framework. This framework is based on the assumption that the width of the Higgs boson is narrow and it is possible to use the zero width approximation. Hence the predicted rate for a given channel can be related to the production cross-section, and the partial and total Higgs boson decay widths in the following way:

$$
\sigma B R(x \rightarrow H \rightarrow f f)=\frac{\sigma_{x} \Gamma_{f f}}{\Gamma_{H}},
$$




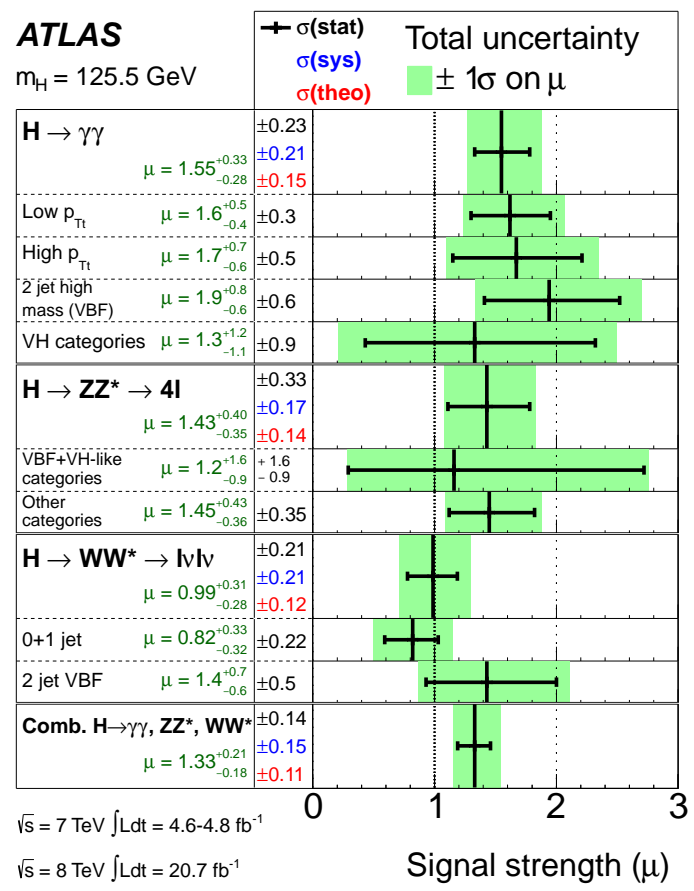

Fig. 7. The measured production strengths for a Higgs boson of mass $m_{H}=125.5$ $\mathrm{GeV}$, normalised to the SM expectations, for di-boson final states and their combination. Results are also given for the main categories of each analysis [1].

where $\sigma_{x}$ is the production cross section through the initial state $x, \mathrm{BR}$ and $\Gamma_{f f}$ are the branching ratio and partial decay width into the final state $f f$, respectively, and $\Gamma_{H}$ the total width of the Higgs boson.

To test the coupling to fermions and bosons, one coupling scale factor is assigned for fermions, $\kappa_{F}$, and one for bosons, $\kappa_{V}$. The results of the fit to the data for the three channels and their combination are shown in Figure 8 . Here, $\kappa_{V}>0$ is assumed, considering that only the relative sign of $\kappa_{F}$ and $\kappa_{V}$ is physical. The two-dimensional compatibility of the SM prediction with the best-fit value is $12 \%$.

The custodial symmetry is tested by measuring the ratio of couplings to the $\mathrm{W}$ and $\mathrm{Z}$ bosons normalised to the SM $\left(\lambda_{W Z}\right)$. The results from fits to the data gives $\lambda_{W Z}=0.82 \pm 0.15$, with a compatibility of the SM prediction with the best-fit value of $20 \%$.

To test the existence of new heavy particles contributing to loop-induced processes, constraints on production and decay loops are extracted by in- 


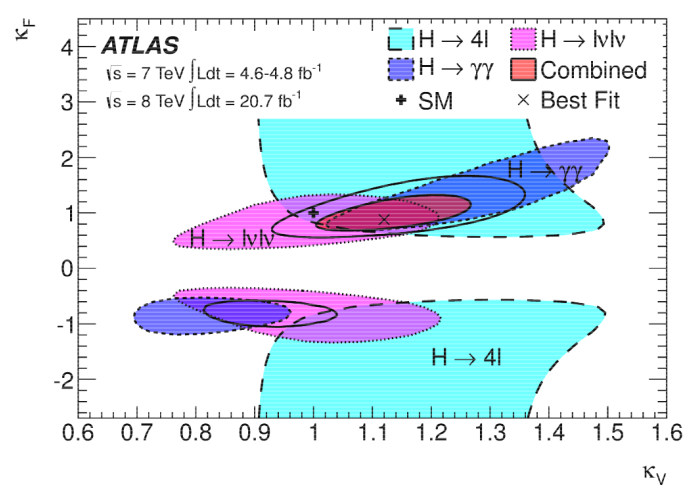

Fig. 8. Likelihood contours $(68 \% \mathrm{CL})$ of the coupling scale factors $\kappa_{F}$. and $\kappa_{V}$ for fermions and bosons, as obtained from fits to the three individual channels and their combination (for the latter, the $95 \% \mathrm{CL}$ contour is also shown). The best-fit result $(\times)$ and the SM expectation $(+)$ are also indicated [1].

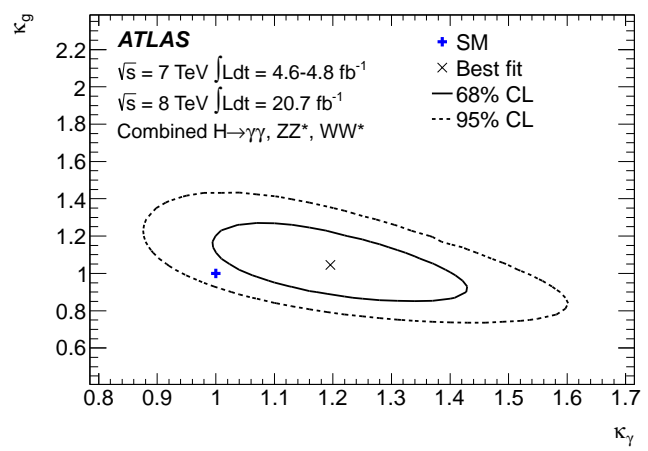

Fig. 9. Likelihood contours as a function of the effective coupling scale factors $\kappa_{F}$ and $\kappa_{V}$, which are the ratios of the effective Higgs couplings to gluons and photons to the SM expectations [1].

troducing effective scale factors $\kappa_{g}$ and $\kappa_{\gamma}$ to parametrise the $g g \rightarrow H$ and $H \rightarrow \gamma \gamma$ loops. Figure 9 show the results of the measurements from a fit to the data. The two-dimensional compatibility of the SM prediction with the best-fit value is $14 \%$.

\subsection{Spin determination}

Studies of the spin and parity quantum numbers of the Higgs boson are performed, using using angular and kinematic distributions for the $H \rightarrow \gamma \gamma$, 


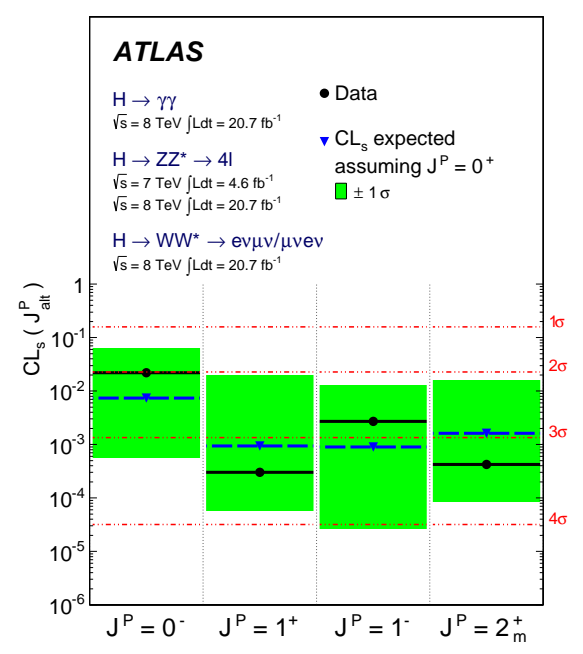

Fig. 10. Expected (blue triangles/dashed lines) and observed (black circles/solid lines) confidence level CLs for alternative spin-parity hypotheses assuming a $0+$ signal. The green band represents the $68 \% \mathrm{CLs}\left(J_{\text {alt }}^{p}\right)$ expected exclusion range for a signal with assumed $0^{+}[3]$.

$H \rightarrow Z Z^{(*)} \rightarrow 4 l$ and $H \rightarrow W W^{(*)}$ decays modes. The data are compatible with the Standard Model $J^{P}=0^{+}$quantum numbers for the Higgs boson, whereas all alternative hypotheses studied, such as $J^{P}=0^{-}, 1^{+}, 1^{-}, 2^{+}$ models, are excluded at confidence levels above $97.8 \%$ [3] as shown in Figure 10 .

\subsection{Differential cross sections measured in the $\gamma \gamma$ channel}

The cross sections are measured using $20.3 \mathrm{fb}^{-1}$ of data at $\sqrt{s}=8 \mathrm{TeV}$, in a dataset with a similar selection to the $H \rightarrow \gamma \gamma$ analysis for the fiducial range of $|\eta|<2.37$ and with $105 \mathrm{GeV}<m_{\gamma \gamma}<160 \mathrm{GeV}$. For each differential measurement, the dataset is divided in bins of different observables such as the transverse momentum $p_{T \gamma \gamma}$ and rapidity $\left|y_{\gamma \gamma}\right|$ of the Higgs boson, the helicity angle $\cos \theta *$ and the jet multiplicity.

For each observable and in every bin, the resonant signal yield is separated from backgrounds using signal plus background fits to the di-photon invariant mass spectrum. These yields are corrected for detector acceptance as well as resolution in the measured observable to determine the fiducial 

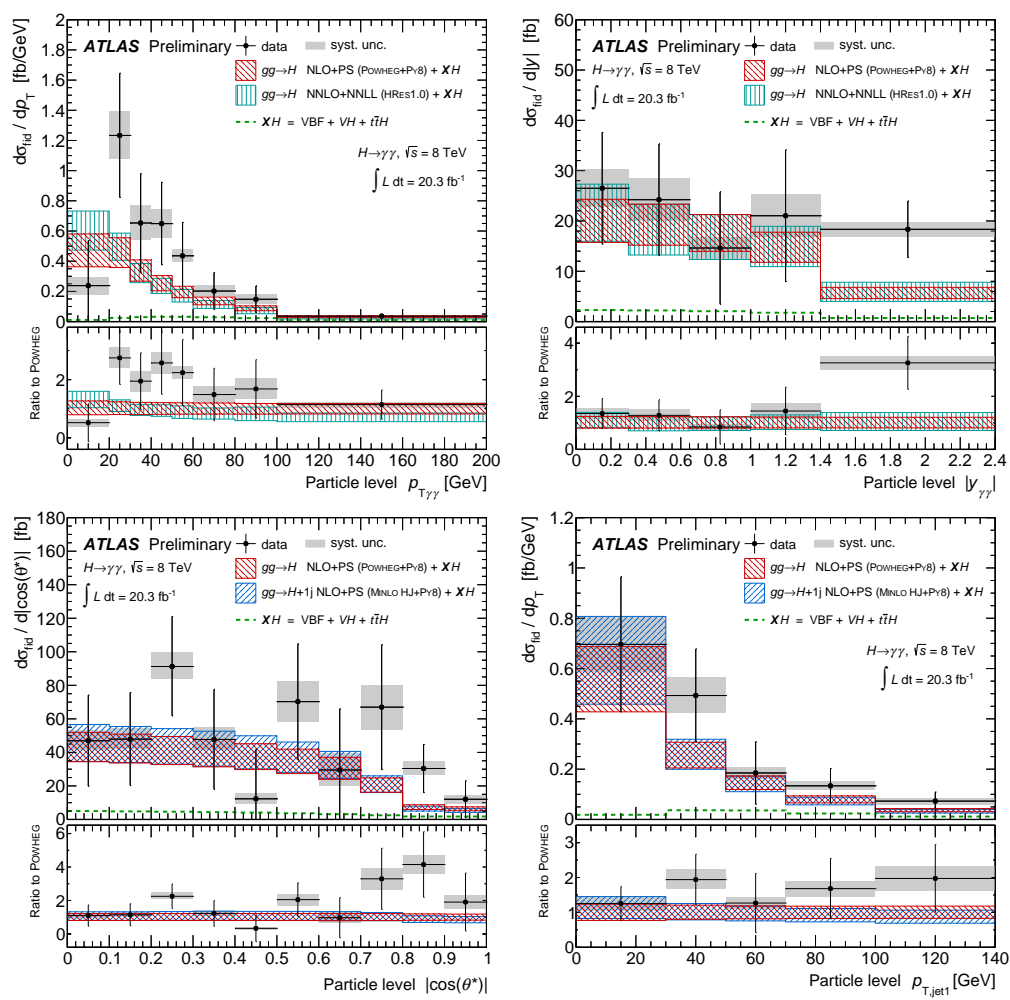

Fig. 11. Observed differential cross sections of the Higgs bosons decaying into two isolated photons, for $\mathrm{pT}$, rapidity, $\cos \theta *$, and leading jet pT. Systematic uncertainties are presented in grey, and the black bars represent the quadratic sum of statistical and systematic errors [4].

differential cross sections.

The unfolded differential cross sections for $p_{\gamma \gamma},\left|y_{\gamma \gamma}\right|, \cos \theta *$ and leading jet pT are shown in Figure 11. The measured differential cross sections are compared with various theoretical predictions. Within the experimental and theoretical uncertainties, no significant deviation from the SM expectation is observed [4].

\section{Rare production and decay modes}

$$
\text { 7.1. } H \rightarrow Z \gamma
$$

The search for the SM Higgs decaying into a $Z \gamma$ is performed using $20.7 \mathrm{fb}^{-1}$ of recorded data at $\sqrt{s}=8 \mathrm{TeV}$ and $4.6 \mathrm{fb}^{-1}$ at $\sqrt{s}=7 \mathrm{TeV}$. No 


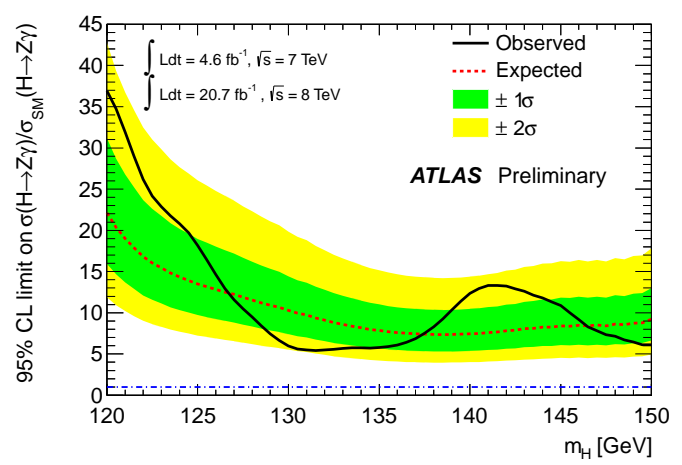

Fig. 12. Observed 95\% CL limits (solid black line) on the production cross section of a SM Higgs boson decaying to $\mathrm{Z} \gamma$, as a function of the Higgs boson mass [5].

significant deviation from the SM prediction is observed and upper limits on the cross section of a Higgs boson with a mass between 120 and $150 \mathrm{GeV}$ are derived (Figure 12). For a Higgs boson mass of $125 \mathrm{GeV}$, the expected and observed limits are 13.5 and 18.2 times the Standard Model, respectively [5].

\section{2. $H \rightarrow \mu \mu$}

The search in the $H \rightarrow \mu \mu$ channel is performed using $20.7 \mathrm{fb}^{-1}$ of data at $\sqrt{s}=8 \mathrm{TeV}$. No evidence of a signal is observed and upper limits are set on the Higgs boson production as a function of the mass. The observed (expected) limit at the 95\% CL for the Higgs boson with a mass of $125 \mathrm{GeV}$ is 9.8 (8.2) times the SM prediction [6] as shown in Figure 13.

$$
\text { 7.3. } p p \rightarrow(V)(H \rightarrow b b)
$$

A search for the $b \bar{b}$ decay of the SM Higgs boson in associated production with a $\mathrm{W}$ or $\mathrm{Z}$ boson is performed using 4.7 and $20.3 \mathrm{fb}^{-1}$ of data at 7 and 8 $\mathrm{TeV}$, respectively. No significant excess is observed above the expected SM backgrounds. For $m_{H}=125 \mathrm{GeV}$, a 95\% CL upper limit of 1.4 times the SM expectation is set on the cross section times branching ratio [7] (Figure 14). 


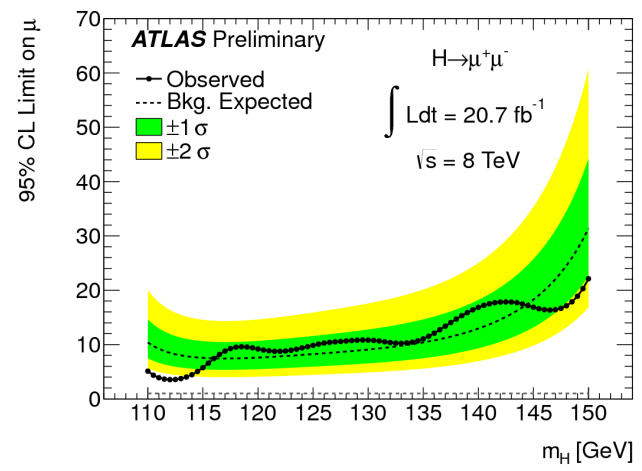

Fig. 13. Observed (solid) and expected (dashed) 95\% CL upper limits on the $H \rightarrow$ $\mu \mu$ signal strength as a function of $m_{H}$ over the mass range $110 \mathrm{GeV}<m_{H}<150$ $\mathrm{GeV}$ [6].

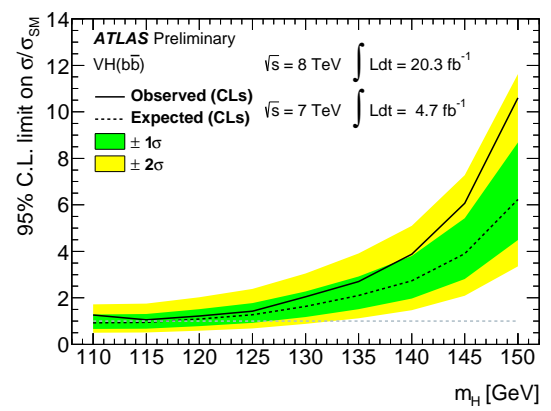

Fig. 14. Expected (dashed) and observed (solid) 95\% CL cross section upper limits, normalised to the SM Higgs boson production cross section, as a function of $m_{H}$ for all channels and data taking periods combined. The expected upper limit is given for the background-only hypothesis [7].

$$
\text { 7.4. } p p \rightarrow(t t)(H \rightarrow \gamma \gamma)
$$

A search for the ttH production mode is performed, using the leptonic and hadronic decay modes of the top quarks and the di-photon decay of the Higgs boson. The analysis uses $20.3 \mathrm{fb}^{-1}$ of proton-proton collisions at $\sqrt{s}=8 \mathrm{TeV}$. No excess over the background prediction is observed and limits are set on the production (see Figure 15). The observed (expected) 95\% confidence level exclusion limits on the $\mathrm{ttH}$ production cross section for a Higgs boson with a mass of $m_{H}=126.8 \mathrm{GeV}$ are 5.3 (6.4) times the 


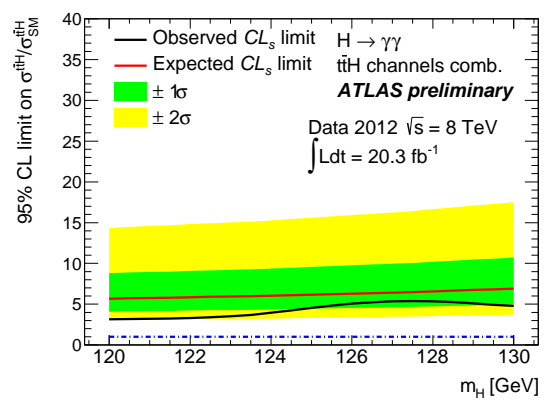

Fig. 15. Observed and expected 95\% CL upper limits on the ttH production cross section [8].

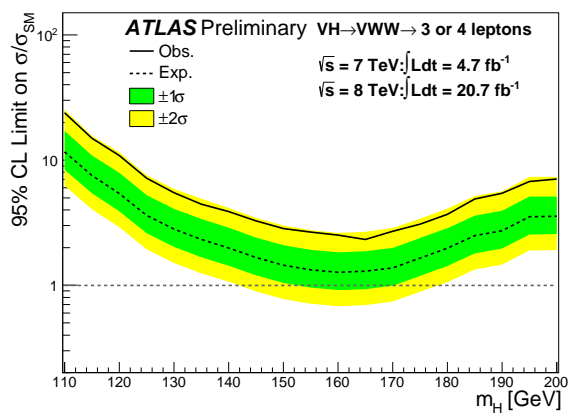

Fig. 16. 95\% CL upper limit on the VH production cross section as a function of the Higgs boson mass, normalised to the SM Higgs boson expectation, from the 3-lepton analysis of the $7 \mathrm{TeV}$ and $8 \mathrm{TeV}$ data and the 4-lepton analysis of the 8 TeV data (full line) [9].

predicted SM values [8].

$$
\text { 7.5. } p p \rightarrow(V)\left(H \rightarrow W W^{(*)}\right)
$$

A search for the VH associated production modes, with $H \rightarrow W W^{(*)} \rightarrow$ $l \nu l \nu$, is performed using $20.7 \mathrm{fb}^{-1}$ of data at $\sqrt{s}=8 \mathrm{TeV}$ and $4.7 \mathrm{fb}^{-1}$ of data recorded at $\sqrt{s}=7 \mathrm{TeV}$. No significant excess is observed over the Standard Model expectations and limits at 95\% CL on the cross section ratio to the SM prediction are set for $110 \mathrm{GeV}<m_{H}<200 \mathrm{GeV}$ (see Figure 16). The observed (expected) limits for a Higgs boson of mass $m_{H}=125 \mathrm{GeV}$ are found to be 7.2 (3.6) times the SM cross section [9]. 

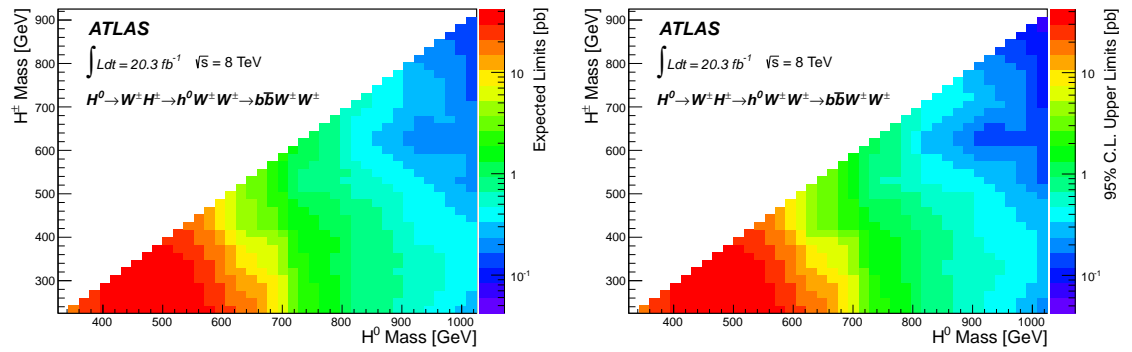

Fig. 17. The expected (top) and observed (middle) 95\% C.L. upper limits on the cross section for $g g \rightarrow H_{0} \rightarrow W H^{p m} \rightarrow W W^{(*)} h \rightarrow W W^{(*)} b b$ as a function of $m\left(H_{0}\right)$ and $\mathrm{m}\left(H^{ \pm}\right)[10]$.

\section{BSM Higgs searches}

\subsection{Search for a multi-Higgs-boson cascade in $W^{+} W^{-} b \bar{b}$ events}

A search for new particles in an extension to the SM that includes a heavy Higgs boson $\left(H_{0}\right)$, an intermediate charged Higgs-boson pair $\left(H^{ \pm}\right)$, and a light Higgs boson $\left(h_{0}\right)$ with a mass of $125 \mathrm{GeV}$, is performed using $20.3 \mathrm{fb}^{-1}$ of $8 \mathrm{TeV}$ data. In the analysis, events involving the production of a single heavy neutral Higgs boson decaying to the charged Higgs boson and a $\mathrm{W}$ boson are searched, where the charged Higgs boson subsequently decays into a $\mathrm{W}$ boson and the lightest neutral Higgs boson decaying to $b \bar{b}$ pair. The data are found to be consistent with Standard Model predictions, and $95 \%$ confidence-level upper limits are set on the product of cross section and branching ratio [10] as shown in Figure 17.

\subsection{Search for $H^{ \pm} \rightarrow \tau \nu+$ jets}

The search for $H^{ \pm} \rightarrow \tau \nu+$ jets with a hadronically decaying $\tau$ lepton in the final state uses $19.5 \mathrm{fb}^{-1}$ of data recorded at $\sqrt{s}=8 \mathrm{TeV}$. No evidence for a charged Higgs boson is found and 95\% CL limits on $B\left(t \rightarrow H^{ \pm} \mathrm{b}\right)$ are set for the mass range $90 \mathrm{GeV}<m\left(H^{ \pm}\right)<160 \mathrm{GeV}$, and for the mass range $180 \mathrm{GeV}<m\left(H^{ \pm}\right)<600 \mathrm{GeV}$, upper limits are set on the production cross section [11], both with the assumption that $B\left(H^{ \pm} \rightarrow \tau \nu\right)=1$ (Figure 18).

\section{Summary}

After the discovery of the new boson, its properties are being measured using the using the full LHC Run I ATLAS data from the $H \rightarrow \gamma \gamma$, $H \rightarrow Z Z^{(*)} \rightarrow 4 l, H \rightarrow W W^{(*)} \rightarrow l \nu l \nu$ decay channels. The combined mass measurement is $m_{H}=125.5 \pm 0.2$ (stat) ${ }_{-0.6}^{+0.5}$ (sys) $\mathrm{GeV}$, the combined 

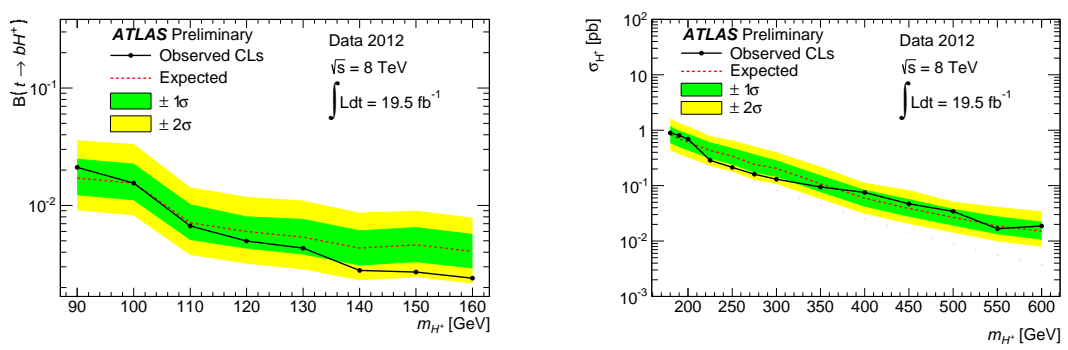

Fig. 18. The expected and observed 95\% CL upper limits for the charged Higgs boson search, with the assumption that $\mathrm{B}\left(H^{ \pm} \rightarrow \tau \nu\right)=1$. Left: For a light charged Higgs bosons, limits on $B\left(t \rightarrow H^{ \pm} b\right)$. Right: Limits on the production cross-section for a heavy charged Higgs [11].

signal strength $\mu=1.33 \pm 0.14$ (stat) \pm 0.15 (sys) , and the spin/parity measurements favour the SM spin $0^{+}$hypothesis.

The first and preliminary results on direct evidence for fermionic decays from $H \rightarrow \tau \tau$, are have been presented where a deviation from the background-only hypothesis is found with an observed (expected) significance of 4.1 (3.2). Furthermore various rare production decay modes and BSM Higgs models searches have been exposed.

The ATLAS experiment is now preparing for LHC Run II, where rare SM Higgs production/decays should achieve observation sensitivity and more precise measurements will be performed to test and challenge the SM predictions.

\section{REFERENCES}

[1] ATLAS Collaboration, "Measurements of Higgs boson production and couplings in diboson final states with the ATLAS detector at the LHC," Physics Letters B, vol. 726, no. 13, pp. 88 - 119, 2013.

[2] ATLAS Collaboration, "Evidence for Higgs boson Decays to the $\tau^{+} \tau^{-}$final state with the ATLAS Detector," Tech. Rep. ATLAS-CONF-2013-108, Nov 2013. https://cds.cern.ch/record/1632191.

[3] ATLAS Collaboration, "Evidence for the spin-0 nature of the Higgs boson using ATLAS data," Physics Letters B, vol. 726, no. 13, pp. 120 - 144, 2013.

[4] ATLAS Collaboration, "Differential cross sections of the Higgs boson measured in the diphoton decay channel using $8 \mathrm{TeV}$ pp collisions," Tech. Rep. ATLAS-CONF-2013-072, Jul 2013. https ://cds . cern. ch/record/1562925. 
[5] ATLAS Collaboration, "Search for the Standard Model Higgs boson in the $H \rightarrow Z \gamma$ decay mode with pp collisions at $\sqrt{s}=7$ and $8 \mathrm{TeV}$," Tech. Rep. ATLAS-CONF-2013-009, Mar 2013. https://cds.cern.ch/record/ 1523683.

[6] ATLAS Collaboration, "Search for a Standard Model Higgs boson in $H \rightarrow \mu \mu$ decays with the ATLAS detector," Tech. Rep. ATLAS-CONF-2013-010, Mar 2013. https://cds. cern. ch/record/1523695.

[7] ATLAS Collaboration, "Search for the bb decay of the Standard Model Higgs boson in associated W/ZH production with the ATLAS detector," Tech. Rep. ATLAS-CONF-2013-079, Jul 2013. https://cds . cern. ch/record/1563235.

[8] ATLAS Collaboration, "Search for ttH production in the $H \rightarrow \gamma \gamma$ channel at sqrt(s) $=8 \mathrm{TeV}$ with the atlas detector," Tech. Rep. ATLAS-CONF-2013-080, Jul 2013. https://cds.cern.ch/record/1564319.

[9] ATLAS Collaboration, "Search for associated production of the Higgs boson in the $W H \rightarrow W W W \rightarrow l \nu l \nu l \nu a n d Z H \rightarrow Z W W \rightarrow l l l \nu l \nu$ channels with the ATLAS detector at the LHC," Tech. Rep. ATLAS-CONF-2013-075, Jul 2013. https://cds. cern.ch/record/1562933.

[10] ATLAS Collaboration, "Search for a multi-Higgs-boson cascade in $W^{+} W b b$ events with the ATLAS detector in pp collisions at $\sqrt{s}=8 \mathrm{TeV}$," Phys.Rev., vol. D89, p. 032002, 2014.

[11] ATLAS Collaboration, "Search for charged higgs bosons in the $\tau+$ jets final state with pp collision data recorded at $\sqrt{s}=8 \mathrm{TeV}$ with the ATLAS experiment," Tech. Rep. ATLAS-CONF-2013-090, Aug 2013. https: //cds. cern. ch/record/1595533. 